\title{
Presentation Title: CASIS Perspective on the Use of the International Space Station National Laboratory for Crystallization Experiments
}

\author{
Authors: Marc Giulianotti and Ken Savin \\ Affiliation: Center for the Advancement of Science in Space (CASIS)
}

\section{Invited Session Title: Crystallization on the International Space Station}

\begin{abstract}
:
The mission of the Center for the Advancement of Science in Space (CASIS) is to maximize the utilization of the International Space Station U. S. National Laboratory (ISS National Lab) for research and technology development aimed at benefitting humankind. CASIS supports collaboration with NASA, other government agencies, not for profit institutions, industry partners, and commercial entities committed to exploring the intellectual, technological and economic opportunities offered by space.
\end{abstract}

CASIS is actively engaged in supporting projects utilizing the ISS National Lab for a variety of life and physical sciences crystallization experiments - organic and inorganic molecules. In addition to supporting current flight projects CASIS is working with the user community to outline the basic science requirements for a long-term crystallization program aboard the ISS National Lab.

This presentation will provide an overview and introduction on the following topics. How to access the ISS National Laboratory platform for all types of crystal growth. Hardware, methodologies, capabilities for microgravity crystallization - organic and inorganic. Physics and chemistry of microgravity crystallography. Results from recent (2015-2018) crystallography experiments aboard the ISS National Laboratory. The potential future for crystallization on the ISS National Laboratory. STEM Outreach Programs for space/ground crystallography. 